\title{
Hydrogen Clouds before Reionization: a Lognormal Model Approach
}

\author{
Hongguang $\mathrm{Bi}^{1,2}$, Li-Zhi Fang ${ }^{2}$, Longlong Feng ${ }^{3,4}$ and Yipeng Jing ${ }^{5,6}$
}

\begin{abstract}
We study the baryonic gas clouds (the IGM) in the universe before the reionization with the lognormal (LN) model which is shown to be dynamcially legitimate in describing the fluctuation evolution in quasilinear as well as nonlinear regimes in recent years. The probability distribution function (PDF) of the mass field in the LN model is long tailed and so plays an important role in rare events, such as the formation of the first generation of baryonic objects. Because in the this model the nonlinear field is directly mapped from the corresponding linear field, we can calculate density and velocity distributions of the IGM at very high spatial resolutions. We simulate the distributions at resolution of $0.15 \mathrm{kpc}$ from $z=7$ to 15 in the LCDM cosmological model. We performed a statistics of the hydrogen clouds at high redshifts, including column densities, clumping factors, sizes, masses, and spatial number density etc. One of our goals is to identify which hydrogen clouds are going to collapse. By inspecting the mass density profile and the velocity profile of clouds, we found that the velocity outflow significantly postpones the collapsing process in less massive clouds, in spite of their masses are larger than the Jeans mass. That indicates that the formation of collapsed clouds with small mass at high redshift is substantial suppressed. Consequently, only massive $\left(>10^{5} \mathrm{M}_{\odot}\right)$ clouds can form objects at higher redshift, and less massive $\left(10^{4}-10^{5} \mathrm{M}_{\odot}\right)$ collapsed objects are formed later. Although the mass fraction in clouds with sizes larger than the Jeans length is already larger
\end{abstract}

${ }^{1}$ Shanghai Astronomical Observatory, Nandan Road 80, Shanghai 200030, China

${ }^{2}$ Department of Physics, University of Arizona, Tucson, AZ 85721

${ }^{3}$ Center for Astrophysics, University of Science and Technology of China, Hefei, Anhui 230026, China

${ }^{4}$ National Astronomical Observatories, Chinese Academy of Science, Chao-Yang District, Beijing 100012, China

${ }^{5}$ Shanghai Astronomical Observatory, the Partner Group of Max-Planck Institut für Astrophysik, Nandan Road 80, Shanghai 200030, China

${ }^{6}$ Beijing Astrophysical Center and Department of Astronomy, Peking University, Beijing 100871, China 
than $1 \%$ at $z=15$, there is only a tiny fraction of mass $\left(10^{-8}\right)$ in the clouds which are collapsed. If all the ionizing photons, and the $\sim 10^{-2}$ metallicity observed at low redshift are produced by the first $1 \%$ mass of collapsed baryonic clouds, the majority of that first generation objects would be happen no much earlier than $z=10$.

Subject headings: cosmology: theory - large-scale structure of the universe

\section{Introduction}

The lognormal (LN) model of the clustering of cosmic mass field was used as a phenomenological description of the density and velocity distributions of the IGM (baryonic gas) in the redshift range $2 \leq z \leq 5$ in the study of the Ly $\alpha$ forest of QSO's absorption spectra (Bi, 1993; Bi, Ge \& Fang 1995; Bi \& Davidsen 1997). Since then the LN model has gained substantial support from dynamical analysis and numerical simulation of the nonlinear evolution of the cosmic mass field. First, it has been found that in the nonlinear regime the dynamics of the growth (irrotational) mode of density perturbations can be sketched by the random-force-driven Burgers' equation (Berera \& Fang 1994, Buchert, Dominguez \& Peres-Mercader 1999). An IGM model based on a random-force-driven Burgers' equation proposed by Jones (1999) yields intermittency of the IGM mass density field, and its probability distribution function (PDF) is found to be lognormal. Numerical simulations have directly shown that a lognormal PDF is a good approximation of the nonlinear density and velocity fields (e.g. Hui, Kofman \& Shandarin, 2000; Yang et al 2001). The LN model can be used to describe the cosmic gravitational clustering not only in quasilinear regimes, but also in highly nonlinear regimes. Recently, a detailed study on the dynamical system consisting of dark matters and the IGM supports also the LN model (Matarrese \& Mohayee 2002).

In this paper, we will use the LN model to study semi-analytically the formation and evolution of the IGM clouds before the reionization. A basic property of the LN model is that the PDF of the mass field is long tailed, and therefore it will play an important role in rare events, such as the formation of the first generation baryonic objects. A number of semi-analytic models have been developed to describe the structure formation in the early universe such as those based on extrapolations of the linear theory (e.g. Couchman \& Rees 1986, Madau, Meikin \& Rees 1997, Tozzi et al. 2000), analytic models (e.g. Tegmark et al. 1997, Miralda-Escude 1998, Valageas \& Silk 1999, Miralda-Escude, Haehnelt \& Rees 2000, Chiu \& Ostriker 2000), the Press-Schechter (PS) formalism (e.g. Haiman \& Loeb 1998), and numerical simulations (e.g. Abel et al. 1998, Bromm, Coppi \& Larson 1999, Gnedin 2000, Cen \& Haiman 2002). See also reviews in Barkana \& Loeb 2001, and Madau 2002. As a 
semi-analytical approach, the non-linear field in the LN model are mapped from the linear density field. Therefore, one can take the advantage to simulate the spatial distributions of density and velocity, so as to calculate the density and velocity profiles of clumpy regions in the distribution, called hydrogen clouds thereafter.

The Jeans length of the IGM is below $1 \mathrm{kpc}$ before the reionization. To study hydrogen clouds on mass scale as small as the Jeans mass $\sim 10^{4} \mathrm{M}_{\odot}$, it is necessary to calculate the IGM distribution on scales at least as fine as $0.2 \mathrm{kpc}$. Using the $\mathrm{LN}$ model, we are able to simulate the IGM distribution on such small scales. We will focus on the abundance of cloud going to collapse. Since the IGM cloud going to collapse is a necessary condition of hosting star formation, the abundance of such collapsing clouds can set effective constrains on the formation of the first generation baryonic objects

The paper is organized as follows. In $\S 2$, we present an updated introduction of the LN model of the clustering of cosmic field. The basic features of the IGM clustering around the epoch of reionization will be discussed in $\S 3$. In $\S 4$, we analyze the statistic properties of the baryonic clumps (hydrogen clouds) based on the simulation of the LN model. In $\S 5$, we identify the clouds which match the condition of going to collapse. Finally in $\S 6$, we summarize the results and our conclusions.

\section{Lognormal model updated}

The basic assumption of the LN model is that the PDF of the density field $\rho(\mathbf{x})$ is lognormal, i.e.

$$
\rho(\mathbf{x})=\overline{\rho_{0}} e^{X(\mathbf{x})}
$$

where $X(\mathbf{x})$ is a Gaussian field with mean $\bar{X}$ and variance $\left[{\overline{(X-\bar{X})^{2}}}^{1 / 2}=\sigma_{0}\right.$. From eq.(1), the mean of $\rho(\mathbf{x})$ is

$$
\bar{\rho}=\overline{\rho_{0}} \exp \left[\bar{X}+\frac{1}{2} \overline{(X-\bar{X})^{2}}\right]
$$

The overall average density $\bar{\rho}$ should always satisfy $\bar{\rho}=\overline{\rho_{0}}$, which is required by mass conservation. Therefore, eq. (2) requires $\bar{X}=-(1 / 2) \overline{(X-\bar{X})^{2}}=-\sigma_{0}^{2} / 2$. The density fluctuation or density contrast of the field is then $\delta=(\rho-1) / \bar{\rho}$.

The PDF of the variable $\rho$ defined by eqs.(1) and (2) is

$$
p(\rho / \bar{\rho})=\frac{1}{(\rho / \bar{\rho}) \sigma_{0} \sqrt{2 \pi}} \exp \left[-\frac{1}{2}\left(\frac{\ln (\rho / \bar{\rho})+\sigma_{0}^{2} / 2}{\sigma_{0}}\right)^{2}\right], \quad \rho \geq 0 .
$$


Obviously, it is normalized as $\int_{0}^{\infty} p(x) d x=1$. The variance of $\rho$ is given by (Vanmarcke 1983)

$$
\sigma=\left[e^{\sigma_{0}^{2}}-1\right]^{1 / 2}
$$

It means the second moment to be $\overline{(\rho / \bar{\rho})^{2}}=\exp \left(\sigma_{0}^{2}\right)$. The high order moments is

$$
\overline{(\rho / \bar{\rho})^{n}}=\exp \left[\left(n^{2}-n\right) \frac{\sigma_{0}^{2}}{2}\right] \text {. }
$$

Equation (5) shows $\left[\overline{\left(\rho / \bar{\rho}^{n}\right.}\right]^{1 / n}>\left[\overline{(\rho / \bar{\rho})^{2}}\right]^{1 / 2}$, i.e. the moment is divergent when $n \rightarrow \infty$. This indicates that the PDF eq.(3) is long tailed. The probability of long tail events $\rho \gg 1$ given by eq.(3) is much larger than that of a Gaussian PDF.

Now we should identify the Gaussian field $X(\mathbf{x})$. When the density perturbation is small, $\delta(\mathbf{x}) \ll 1$, the mass field should be in linear regime $\delta_{0}(\mathbf{x})$, i.e. we need $\delta(\mathbf{x}) \simeq \delta_{0}(\mathbf{x})$. This yields $X=\delta_{0}(\mathbf{x})-\sigma_{0}^{2} / 2$, or

$$
\rho(\mathbf{x})=\bar{\rho}_{0} \exp \left[\delta_{0}(\mathbf{x})-\sigma_{0}^{2} / 2\right]
$$

where $\sigma_{0}=\left\langle\delta_{0}^{2}\right\rangle^{1 / 2}$ is the variance of the linear Gaussian field of $\delta_{0}(\mathbf{x})$ on the scale $R$ considered. When $\sigma_{0}$ is small, eq.(4) gives $\sigma=\sigma_{0}$. Equation (6) is a basic assumption of the LN model. With eq.(6), the nonlinear mass field, $\rho(\mathbf{x})$, is given by an exponential mapping from the corresponding linear density field $\rho_{0}(\mathbf{x})$ or $\delta_{0}(\mathbf{x})$.

The first successful application of the LN model mapping eq.(6) is to model the IGM distribution observed by the QSO Ly- $\alpha$ forests (Bi 1993 and Bi \& Davidsen 1997). However, the LN model is not only useful in weakly nonlinear regime, but also for highly nonlinear evolution. The dynamical study of the lognormal PDF can be traced back to the adhesion approach, which sketches the nonlinear evolution of the growth mode of the cosmic gravitational clustering with the Burgers' equation (Gurbatov, Saichev \& Shandarin 1989). This equation yields reasonable mass function of clumps (Vergassola et al. 1994). Considering the stochastic nature of field variables, the cosmic clustering actually should be described by the random-force-driven Burgers' equation (Berera \& Fang 1994; Buchert, Dominguez \& Peres-Mercader 1999). On the other hand, the lognormal PDF is found to be good approximation of density field described by this equations. Therefore, the log-normal model is dynamically legitimate to describe the cosmic clustering in quasilinear as well as nonlinear regimes. This is also supported by numerical simulation. The one-point distribution of the cosmic mass and velocity fields on nonlinear regime, such as the number density of galaxies, pairwise velocity, angular momentum, etc from observed data and simulation samples, are in good agreement with lognormal distribution (e.g. Kofman et al. 1994, Hui, Kofman, \& Shandarin 2000, Yang et al 2001, Pando, et al 2002). 


\section{IGM distribution with lognormal model}

\subsection{Jeans length of baryonic matter}

In a homogeneous universe with the mean mass density $\bar{\rho}$, the Jean length of gaseous baryonic matter or the IGM is defined by $\lambda_{b} \equiv v_{s}(\pi / G \bar{\rho})^{1 / 2}$, where $v_{s}$ is the sound speed of the gas. For the current study, it is convenient to use a comoving scale $x_{b}$ given by

$$
x_{b} \equiv \frac{1}{H_{0}}\left[\frac{2 \gamma k_{B} T_{m}}{3 \mu m_{p} \Omega(1+z)}\right]^{1 / 2},
$$

which is $2 \pi$ times smaller than $\lambda_{b}$. In eq.(7), $T_{m}$ and $\mu$ are the mean temperature and molecular weight of the gas, $\Omega$ is the cosmological density parameter of total mass and $\gamma$ the ratio of specific heats. The corresponding Jeans mass is $m_{J}=(4 \pi / 3) \lambda_{b}^{3} \bar{\rho}$.

Primordial baryons, created at the time of nucleosynthesis, recombines with electrons to become neutral gas at $z \sim 1000$, which corresponds to the Jeans mass of about $10^{6} M_{\odot}$. Before $z=137$, the residual ionization of the cosmic gas keeps its temperature locked to the CMB temperature. After that the gas cools down adiabatically during expanding of the universe. Assuming $\gamma=5 / 3$, i.e. hydrogen temperature $T \propto \rho_{b}^{2 / 3}$, where $\rho_{b}$ is the mean mass density of baryonic matter, the evolution of the comoving Jeans length will depend approximately on $(1+z)$. At $z=10$, the hydrogen temperature is $\sim 1.8 \mathrm{~K}$ (Medvigy \& Loeb 1991), the Jeans length $x_{b} \sim 1 \mathrm{kpc}$, and the Jeans mass drops to about $10^{4} M_{\odot}$. Note that the Jeans mass is 6 magnitudes smaller than that of galaxies we observed. Figure 1 plots the comoving Jeans length $x_{b}$ as a function of the cosmic scale factor $1 /(1+z)$.

During the reionization, the baryonic gas will be heated by UV ionizing background with temperature from $1.8 \mathrm{~K}$ to $\sim 1.3 \times 10^{4} \mathrm{~K}$; and there is a 2 magnitudes increasing in $x_{b}$ or 6 magnitudes in the Jeans mass (e.g. Ostriker \& Gnedin 1996). In Fig. 1, the reionization is assumed to happen at $z=7$ instantly, i.e. the IGM is assumed to be almost completely neutral and ionized before and after the reionization, respectively. It should be emphasized that the assumption of reionization redshift $z=7$ does not affect on the result of clustering before reionization discussed below. That is, for instance, the calculation of clustering at $z=12$ is independent of whether the reionization redshift is at 7 or 10 .

After the reionization, the IGM temperature is maintained at about $\sim 10^{4} \mathrm{~K}$ by the UV background photons, and therefore, $x_{b}$ will gradually increase with the decrease of $z$ due to the factor $(1+z)$ in eq. $(1)$. Note that the HeII ionization occurs at redshift 3.3 that leads

to an increase of the IGM temperature from $1.3 \times 10^{4} \mathrm{~K}$ before $z=3.3$ to $2.5 \times 10^{4} \mathrm{~K}$ after $z=3.3$ (Theuns et al 2002). This is shown in Fig. 1 by the small jump of $x_{b}$ at the redshift $\sim 3.3$ 


\subsection{IGM mass density field in lognormal model}

The linear evolution of the IGM mass field, $\rho_{0}(\mathbf{x})$, driven by the gravity of dark matter mass field, $\rho_{d m}(\mathbf{x})$, has been studied by using various assumptions of the thermal property of the IGM (e.g. Nusser 2000, Matarrese \& Mohayee 2002). A common conclusion is

$$
\delta_{0}(\mathbf{k}, t)=(1+\text { decaying terms }) \delta_{d m}(\mathbf{k}, t)+\text { decaying terms } \quad \text { if } k \ll k_{J} \text {. }
$$

where $\delta_{0}(\mathbf{k}, t)$ and $\delta_{d m}(\mathbf{k}, t)$ are, respectively, the Fourier transform of density fluctuations of the IGM $\delta_{0}(\mathbf{x})=\left[\rho_{0}(\mathbf{x}) / \bar{\rho}_{0}\right]-1$ and the dark matter, $\delta_{d m}(\mathbf{x})=\left[\rho_{d m}(\mathbf{x}) / \bar{\rho}_{d m}\right]-1$. The decaying terms in Eq.(10) depend on the initial conditions. That is, regardless specific assumptions of thermal processes and initial condition, the linear fluctuations of the IGM mass field on scales larger than the Jeans length $x_{b}$ always follows the dark matter mass field (Fang et al 1993)

$$
\delta_{0}(\mathbf{x})=\frac{1}{4 \pi x_{b}^{2}} \int \frac{\delta_{d m}\left(\mathbf{x}_{1}\right)}{\left|\mathbf{x}-\mathbf{x}_{1}\right|} e^{-\frac{\left|\mathbf{x}-\mathbf{x}_{1}\right|}{x_{b}}} d \mathbf{x}_{1}
$$

Obviously, $\left\langle\delta_{0}\right\rangle=\left\langle\delta_{d m}\right\rangle=0$.

With the LN model of $\S 2$, the nonlinear mass field of the IGM, $\rho_{b}(\mathbf{x})$, has to be is given by an exponential mapping from the corresponding linear density field of dark matter $\rho_{0}(\mathbf{x})$ as

$$
\rho_{b}(\mathbf{x})=\bar{\rho}_{b} \exp \left[\delta_{0}(\mathbf{x})-\sigma_{0}^{2} / 2\right]
$$

where $\sigma_{0}$ is the variance of the linear Gaussian field of $\delta_{0}(\mathbf{x})$ on the scale of the Jeans length. To simplify eq.(12), we use normalized the mean baryonic matter density $\bar{\rho}_{b}=1$ below.

The number of $\sigma_{0}$ is shown in Fig. 2, in which, we use the low density flat cold dark matter model (LCDM) with the density parameter $\Omega_{0}=0.3$, the cosmological constant $\Omega_{\Lambda}=0.7$ and the Hubble constant $h=0.7$. The linear power spectrum $P(k)$ is given by the fitting formula given by Eisenstein \& $\mathrm{Hu}$ (1998). The linearly increasing of $\sigma_{0}$ with cosmic factor $1 /(1+z)$ is due to the linear increasing of the density perturbations. The variance $\sigma$ of the LN PDF [eq.(3)] is also plotted in Fig. 2. We see from Fig. 2 that the first time of the variance $\sigma_{0}$ reaching to order of one is in the period of redshift $15>z>7$. This should be the epoch of the first generation collapsed object formation.

When the fluctuations $\delta_{0}(\mathbf{x})$ are small, $\delta_{0}(\mathbf{x}) \ll 1$, eq.(10) yields $\delta(\mathbf{x}) \simeq \delta_{0}(\mathbf{x})$. It is the linear solution eq.(8). On the other hand, on scales $x \leq x_{b}$, eq.(10) gives the nonlinear relation between the IGM density distribution and the dark matter gravitational potential as

$$
\rho_{b}(\mathbf{x}) \propto \exp \left[-\frac{\mu m_{p}}{\gamma k T} \psi_{d m}(\mathbf{x})\right] .
$$


Eq.(11) is the well-known isothermal hydrostatic solution, which describes highly clumped structures such as intracluster gas (Sarazin \& Bahcall 1977). Therefore, the density distribution $\rho_{b}(\mathbf{x})$ in eq.(10) is consistent with highly nonlinear distribution of the baryonic mass in the dark matter gravitational potential wells. Note that the primordial potential $\psi_{d m}$ remain linear and Gaussian much longer time than the density (Brainerd, Scherrer \& Villumsen 1983; Bagla \& Padmanabhan 1994), eq.(11) directly shows that the IGM field $\rho_{b}(\mathbf{x})$ is lognormal. Thus, if we require that the mapping between the IGM field $\rho_{b}(\mathbf{x})$ and the linear dark matter field $\delta_{d m}(\mathbf{x})$ should satisfy 1.) the linear relation eq.(8) in linear regime; 2.) the exponential relation eq.(11) in highly nonlinear regime, the mapping of eqs.(10) probably is the most reasonable one. Therefore, the lognormal mapping eq.(10) can uniformally describe the IGM distribution from linear, weakly nonlinear, to highly nonlinear regimes, corresponding to density contrast $\ll 1, \simeq 1$, and $\gg 1$ respectively, without introducing extra parameters before the dynamics of clouds are dominated by hydro processes, such as cooling and heating during star formation.

The LN model eq.(9) - (10) is successful to fit the transmitted flux of the QSO Ly $\alpha$ absorption spectrum (Bi \& Davidsen 1997; Feng \& Fang, 2001). The lognormal PDF of the IGM field also gives better fitting to the recent detected intermittency of the transmitted flux with high resolution, high signal to noise ratio samples of QSO Ly $\alpha$ absorption spectra (Jamkhedkar, Zhan, \& Fang, 2000, Zhan, Jamkhedkar, \& Fang, 2001, Feng, Pando, \& Fang 2001, Feng, Pando \& Fang 2003, Jamkhedkar et al. 2003).

\subsection{Volume filling factor and cumulative mass fraction of IGM clouds}

We now study the formation and collapsing of overdense hydrogen clouds in the IGM field with the lognormal mapping. First we demonstrate the lognormal PDF with the volume filling factor $V(>\rho)$, which is the fractions of volume with density larger than a given $\rho$. From eqs.(3) or (10) we have

$$
V(>\rho)=\int_{\rho}^{\infty} p(\rho) d \rho=\frac{1}{2} \operatorname{erfc}\left(\frac{\sigma_{0}}{2 \sqrt{2}}+\frac{\ln \rho}{\sqrt{2} \sigma_{0}}\right) .
$$

Figure 3 shows $V(>\rho)$ for $\sigma_{0}$ on the Jeans length scales at redshifts $z=30,20,10$ and 7 . For a uniform Gaussian random field, roughly we have half space volume with density lower than the mean $\rho=1$, and half larger than $\rho=1$. However, Fig. 3 shows that $V(>1)<1 / 2$ even when $z=30$. That is, the mass field of the IGM has already deviated from a Gaussian field at very early time. Fig. 3 shows also that most IGM has fallen into clumps and most space is occupied by very low density gas. This is why the lognormal model is able to fit intermittent fields (Pando, et al. 2002; Feng, Pando \& Fang, 2003). In these fields, the mass 
is concentrated in peaks or spikes, which are randomly and widely scattered in space with a low mass density surrounded.

The long tail of the LN model can more easily be seen with the cumulative mass fraction $M(>\rho)$, which is the fraction of mass in regions having mass density larger than a given $\rho$, given by

$$
M[>(\rho / \bar{\rho})]=\int_{\rho / \bar{\rho}}^{\infty} x p(x) d x=\frac{1}{2} \operatorname{erfc}\left(\frac{\ln (\rho / \bar{\rho})}{\sqrt{2} \sigma_{0}}-\frac{\sigma_{0}}{2 \sqrt{2}}\right) .
$$

Figure 3 shows $M(>\rho)$ for $\sigma_{0}$ on the Jeans length scales at redshifts $z=30,20,10$ and 7. The curves of $M(>\rho)$ at high $\rho$ shows clearly the long tail. For instance, for the curve of $z=7$, the number of $M(>100)$ is less $M(>10)$ only by a factor of about 10 . That is, the mass fraction of large mass events $(\rho=100)$ in $R$ can be $10 \%$ of small mass events $(\rho=10)$. This is because that the variance $\sigma_{0}$ at $z=7$ is about 1.8 . Objects of $\rho=10$ correspond to $\ln \rho / 1.8 \simeq 1.3 \sigma_{0}$ event, and $\rho=100$ to $\ln \rho / 1.8 \simeq 2.6 \sigma_{0}$ event. Therefore, the probabilities for $\rho=10$ and $\rho=100$ are different only by a factor about 10 . On the other hand, if the PDF is Gaussian, $\rho=10$ objects corresponds to 1.3- $\sigma_{0}$ event, while high density $\rho=100$ corresponds to $7.7-\sigma_{0}$ event. In this case, the number of $\rho=100$ objects is completely negligible with respect to $\rho=10$ objects.

Figure 3 shows that $M(>\rho)$ is significant for $\rho=10$ and $z>10$. On the other hand, we have always $V(>\rho) \sim 0$ with $\rho=10$, regardless redshift. This indicates that a significant part of mass concentrates in a small volume. That is dense objects at high redshifts. The long tail events may not be important at low redshift, as at that time, $\sigma_{0}$ on large scales is close to 1 , and the formation of large mass objects is no longer to be rare events.

\section{Statistics of Hydrogen Clouds}

\subsection{Simulations of the IGM distribution}

To study the clustering and collapsing of hydrogen clouds, we produce simulation samples of spatial distribution of gas $\rho(\mathbf{x})$ with the LN model developed in last section. In order to quickly grasp the features of these distributions, we will simulate 1-D distribution. The details of the simulation procedure has been given in Bi \& Davidsen (1997). A brief description is as follows.

We first simulate the 1-D density and velocity distribution in the Fourier space, $\delta_{0}(k)$ and $v(k)$, which are two Gaussian random fields. Both $\delta_{0}(k)$ and $v(k)$ are given by the power 
spectrum, $P_{0}(k)$, as follows (Bi 1993; Bi, Ge \& Fang 1995)

$$
\begin{aligned}
& \delta_{0}(k, z)=D(z)(u(k)+w(k)), \\
& v(k, z)=F(z) \frac{H_{0}}{c} i k \alpha(k) w(k),
\end{aligned}
$$

where $D(z)$ and $F(z)$ are the linear growth factors for fields $\delta_{0}(x)$ and $v(x)$ at redshift $z$. The fields $w(k)$ and $u(k)$ are Gaussian with power spectra given by

$$
\begin{gathered}
P_{w}(k)=\alpha^{-1} \int_{k}^{\infty} P_{0}(q) 2 \pi q^{-1} d q, \\
P_{u}(k)=\int_{k}^{\infty} P_{0}(q) 2 \pi q d q-P_{w}(k),
\end{gathered}
$$

where $P_{0}(k)$ is the power spectrum of the 3 -D field $\delta_{0}(\mathbf{x})$. Functions $\alpha(k)$ in eq.(16) is defined by

$$
\alpha(k)=\frac{\int_{k}^{\infty} P_{0}(q) q^{-3} d q}{\int_{k}^{\infty} P_{0}(q) q^{-1} d q}
$$

From eq.(9), we have

$$
P_{0}(k)=\frac{P_{d m}(k)}{\left(1+x_{b}^{2} k^{2}\right)^{2}}
$$

where $P_{d m}(k)$ is the dark matter power spectrum in 3-D. Thus, for a given $P_{d m}(k)$ and $x_{b}$, one can produce the distributions $\delta_{0}(k)$ at grid points $k_{i}, i=1,2, \ldots, N$ in the Fourier space. The spatial distributions in the real line-of-sight space $\delta_{0}(x)$ can be obtained by using a Fast Fourier Transform. Since the velocity follows the linear evolution longer than the density, we can use the linear $v(k)$ and its Fourier counterpart as velocity field.

The Jeans length before the reionization is about one $\mathrm{h}^{-1} \mathrm{kpc}$. It requires the resolution of simulation to be less than $0.2 \mathrm{kpc}$. Our simulation range is $40 \mathrm{Mpc}$ in comoving space. The total number of pixels is 262144 , so the pixel size is $0.152588 \mathrm{kpc}$. Three random samples of the density field with 1 Mpc comoving size are plotted for $z=15,10$ and 7 in Fig. 4. The prominent spikes correspond to the dense hydrogen clouds which are candidates of the collapsing objects. Such hydrogen clouds occur not only at redshift $z=7$, but also at $z=15$. Although the number and height of the spikes decrease with higher redshifts, this $z$-dependence actually is not very sharp. The typical size of the clouds shown in Fig. 4 is of the Jeans length, but they have different height. This indicates that the probability of the spike events on the same size is not sharply dependent on the their height, i.e. the probability does not sharply depend on the mass density $\rho$ of the spikes, but only on $\ln \rho$. This is an effect of the lognormal long tail. 


\subsection{Basic properties of hydrogen clouds}

To identify hydrogen clouds, we first smooth the simulated density field with a Gaussian filter of dispersion $x_{b}$. With the smoothed field, baryonic or hydrogen clouds are identified as the regions between two successive minima in the field. For each clouds, we have a mass density profile between the two minima. The maximum between the two successive minima is the central position of the cloud. The width $D$ is defined to be the FWHM of the top density. The column density $N_{H I}$ of an absorber is obtained by summing gas densities in each pixel from the first minimum to the next minimum in the smoothed field. For each cloud, we assign a peculiar velocity to be the velocity at the top density, and the internal velocity profile is the peculiar velocity of each pixel relative to the center velocity.

Since column density depends on both hydrogen number density and the size of clouds, it may not be a good indicator for the density contrast of hydrogen clouds. For instance, two clouds with the same column density may have 10 times difference in their density contrast due to a 10 times difference of their sizes. If such cases are common, one can not measure mass density with the column density. However, statistically we have a good reason to use column density $N_{H I}$ to characterize the mass density of baryonic clouds. Fig. 5 plots the relation between the column density $N_{H I}$ and density $\rho$ of clouds identified from one realization of the 1-D field. Fig. 5 shows a tight correlation between the column density $N_{H I}$ and the mass density $\rho$ of clouds. For a given $N_{H I}$, the dispersion of $\rho$ is no larger than $20 \%$. That is, the column density is mainly determined by the cloud mass density, not their size. A similar correlation has also been found among the pre-collapsed halos identified from N-body simulation samples (Xu, Fang \& Wu 2000). Thus, the IGM clustering in dark age can be approximately described by the statistics of the hydrogen clouds with the number $N_{H I}$.

We first calculate $N_{c}\left(>N_{H I}, z\right) \mathrm{h} \mathrm{Mpc}^{-1}$, which is the 1-D comoving number density of clouds with column densities larger than a given $N_{H I}$. The redshifts are taken to be $z=7$, 10 and 15. The differential number density is $d N_{c}\left(>N_{H I}, z\right) / d \ln \left(N_{H I}\right)$, which is plotted in Figure 6a. We can see from Fig. 6 a that clouds with $N_{H I} \geq 10^{20}$ at $z=7$ are much more than that at $z=15$. It indicates that most clouds $N_{H I} \geq 10^{20}$ formed at redshifts less than 15 .

Figure $6 \mathrm{~b}$ presents the cumulated mass fraction of clouds with column density larger than a given $N_{H I}$. It also shows that mass fraction of clouds with $N_{H I}>10^{20.5}$ underwent a significant evolution from $z=15$ to 7 . The mass fraction of $N_{H I}>10^{20}$ clouds at $z=15$ is about $1 \%$, but it is about $10 \%$ at $z=7$. For clouds with column densities $10^{19.0}<N_{H I}<10^{19.75}$, the number density and mass fraction at redshifts 7 to 15 are comparable. 
Figures $6 \mathrm{c}$ and $6 \mathrm{~d}$ give, respectively, the mean top density and the mean comoving width $D$ of $N_{H I}$ clouds. Figures $6 \mathrm{c}$ and $6 \mathrm{~d}$ show that the clouds with $N_{H I} \geq 10^{20}$ at $z=7$ have smaller size (width) and higher central density than that at $z=15$ and 10 . This shows that hydrogen clouds in collapsing in the period from $z=15$ to $z=7$. The mass density of $N_{H I} \geq 10^{19.25}$ clouds has overdensity $\rho>5$ at all redshifts. It means that the $N_{H I} \geq 10^{19.25}$ clouds were already going to collapse as early as $z=15$.

To study the 3-D statistics of the clouds, we assume that most clouds are spherical in the 3 -D space with scale $D$. This is equal to approximate the non-linear dynamical evolution of a clouds as a spherical collapsing process. This approximation probably is poor for clouds with low $N_{H I}$, but would be better for higher $N_{H I}$. Thus, we can estimate the baryonic mass of the cloud by $\rho m_{p} D^{3}$. The result is plotted in Figure $7 \mathrm{a}$. We see that the $M-N_{H I}$ relation is insensitive to redshift. Fig. $7 \mathrm{~b}$ is the cumulated mass fraction of 3 - $\mathrm{D}$ clouds with mass larger than a given $M$. It is interested to see that the mass fraction of clouds with mass in the range $M<10^{4.5} \mathrm{M}_{\odot}$ is weakly dependent on redshift from $z=7$ to 15 . The mass fraction for $M \geq 10^{4.5} \mathrm{M}_{\odot}$ is significantly dependent on redshift.

Figure $8 \mathrm{a}$ is the 3-D cumulative number density of clouds with column density larger than a given $N_{H I}$. Figure $8 \mathrm{~b}$ is similar to Figure $8 \mathrm{a}$, but it is with respect to the mass $M$ of clouds. We see again that the cumulative number densities of clouds at $N_{H I} \simeq 10^{19.25}$ or $M \simeq 10^{4.5} \mathrm{M}_{\odot}$ are not a strong function of redshifts from 7 to 15 . The cumulative number densities of massive clumps $N_{H I}>10^{20}$ or $M>10^{5} \mathrm{M}_{\odot}$ decreases with higher redshift quickly. From these statistics, we can conclusion that, in the LN model, the baryonic clouds with mass $M>10^{5} \mathrm{M}_{\odot}$ underwent a strong evolution in the epoch of $15>z>7$. But for clouds with mass $M<10^{4.5} \mathrm{M}_{\odot}$, the evolution is moderate.

\section{Clouds going to collapsing}

\subsection{Density profile and collapsing}

We now identify which clouds are going to collapse. Obviously collapsed IGM clouds is a necessary condition for hosting star formation. To study the details of the collapsing, we will calculate the density profile within each cloud. The mean densities profile of clouds along the line-of-sight have been plotted in Fig. 9, in which the horizontal axis is the comoving distance from the center of the clouds, and redshifts are taken to be 15, 12, 10 and 7 . For each redshift, the successive curves from lower to higher correspond to column density $\log N_{H I}=16.25+m \times 0.5$, and $m=0,1, \ldots 8$.

Fig. 9 shows that all density profiles are approximately exponential, i.e. $\rho \propto \exp \left(-x / 2 x_{0}\right)$, 
where $x_{0}$ is of the order of $x_{b}$. The exponential profile comes from the exponential factor in eq.(11). In Fig. 9, we can see that the comoving density profile of clouds with $N_{H I}=10^{17.75}$ is almost independent of redshift $z=15$ to 7 . That is, the comoving size of clouds of $N_{H I}=10^{17.75}$ is almost independent of redshift, and therefore, they are expanding with the Hubble flow. These clouds are comoving with the Hubble expansion. This is expected. From Fig. 5, we seen that $N_{H I}=10^{17.75}$ corresponds to about $\rho=1$, i.e. these "clouds" have the same density as background universe. It should follow the Hubble expansion.

For clouds with $N_{H I}<10^{17.75}$, or $\rho<1$, the comoving size is bigger with smaller redshift. In other words, these "clouds" actually are in voids. They are a part of the voids. Therefore, their expansion is faster than Hubble streaming.

For clouds with $N_{H I} \geq 10^{18.25}$ the comoving size is smaller for smaller redshifts. This is, there are in the phase of turn around. The mean density of these clouds at $z=7$ is $\rho \sim 6$ (Fig. 6). These clouds have decoupled from the Hubble expansion. Their expansion is slower than the Hubble expansion. However, we should not simply identify all clouds with $N_{H I} \geq 10^{18.25}$ to be in collapsing, because they may still be in physical expanding, but only the expanding velocity is less than the Hubble velocity.

\subsection{Velocity profile and collapsing}

Whether a cloud is collapsing should also be investigated through its velocity profile. We calculate the peculiar velocity profile for all clouds identified from the 3,000 realizations of 1-D IGM field. The mean 1-D velocity profiles for various $N_{H I}$ clouds are plotted in Fig. 10, in which the horizontal axis is the comoving distance from the center of cloud, and velocity is measured with respect to the center of clouds. The redshifts are taken to be 15, 12, 10 and 7. For each redshift, the successive curves from smaller to larger velocity correspond to column density $\log N_{H I}=16.25+m \times 0.5$, and $m=0,1, \ldots 8$.

Figure 10 also shows the Hubble flow $v_{H}$ with respect to the center of the clouds. The dotted lines of Fig. 10 actually is $-v_{H}$. The physical velocity profiles is then given by $v+v_{H}=v-\left(-v_{H}\right)$. Fig. 11 is the same as Fig. 10, but shows a zoom-in profile of the central parts of the clouds.

In Figs. 10 and 11, we can classify baryonic clouds into three types. 1.) velocity profile of $v$ is negative, $v<0$, on the side of separation $<0$ and positive, $v>0$, on the side of separation $>0$. These clouds are expanding away from their centers, i.e. their physical expansion velocity is faster than the Hubble flow. 2.) the velocity profile of $v$ is positive, $v>0$, on the side of separation $<0$ and negative, $v<0$, on the side of separation $>0$, 
but the physical velocity profile is negative, $v+v_{H}<0$, on the side of separation $<0$ and positive, $v+v_{H}>0$, on the side of separation $>0$. These clouds are decoupled from the Hubble flow, but they still remain in expanding phase, and not reached yet to their maximum expansion. 3.) the physical velocity profile is positive, $v+v_{H} \geq 0$, on the side of separation $<0$ and negative, $v+v_{H} \leq 0$, on the side of separation $>0$. These clouds are in the phase of maximum expansion, or starting to collapse. In other words, its infall velocity is greater than the Hubble velocity in physical space.

Obviously, only clouds of type (3) can be identified as collapsing or collapsed clouds. That is, in Figs. 10 and 11, only the $N_{H I}$ clouds having the $v$ velocity profiles (solid line) equal to or higher than the negative Hubble flow $-v_{H}$ (dotted line) are the possible spots of star formation. Therefore, not all clouds with size larger than the Jeans length are in the collapsing phase. Although clouds with small $N_{H I}$ have size larger than the Jeans length, the velocity outflow of clouds significantly postpones their collapsing. This leads to a suppression of formation of low mass collapsed clouds in high redshifts. This result is consistent with the simulation result (Gnedin, 2000), which shows that the formation of low mass objects in the redshift range $12>z>7$ is significantly suppressed with respect to the Jeans length prediction.

\subsection{Constrains on the first star formation}

Besides the Jeans length of IGM in eq.(9), we did not introduced any other parameters related to the hydro processes of star formation in this paper. Even so, we can already set some constrains on the formation of first generation stars by considering that collapsed clouds are the necessary environment of hosting star formation.

As mentioned in last subsection, only clouds of type (3) are collapsed or collapsing, and can play the role of the host of star formation. Thus, the first generation IGM clustering can be seen from the properties of the type (3) clouds, and their redshift-dependence. Table 1 lists the basic properties of these clouds, including the minimum column densities $\left(\log N_{H I}\right)_{\min }$, the minimum mass $M_{\min }\left(\mathrm{M}_{\odot}\right)$ and the minimum density $\rho_{\min }$. All the minimum density $\rho_{\text {min }}$ of the collapsing clouds are $>10$ or even $>>10$. Note $\sigma_{0}$ is of order of one, the events of $\rho_{\min }>10$ are really rare, or very rare. Therefore, the first generation of star formation rely on the long tail of the PDF.

Figure 12 shows the redshift dependence of the cumulative mass fraction $M\left(>M_{J}\right)$ of clouds larger than Jeans mass, and $M\left(>\rho_{\text {min }}\right)$ of clouds in collapsing and collapsed baryonic objects. One can clearly see from Fig. 12 that the collapsing of hydrogen clouds is 
Table 1: Properties of collapsing clouds vs. redshift $z$

\begin{tabular}{lllll}
\hline redshift & 15 & 12 & 10 & 7 \\
\hline$\left(\log N_{H I}\right)_{\min }$ & 22.25 & 20.25 & 19.5 & 18.75 \\
$M_{\min }\left(\mathrm{M}_{\odot}\right)$ & $10^{6.5}$ & $10^{5.3}$ & $10^{4.4}$ & $10^{3.5}$ \\
$\rho_{\min }$ & $3.1 \times 10^{3}$ & $1.9 \times 10^{2}$ & 35 & 19 \\
\hline
\end{tabular}

significantly suppressed when $z>12$. The evolution of $M\left(>M_{J}\right)$ is moderate in the redshift range of $7<z<15$, while the redshift-dependence of $M\left(>\rho_{\min }\right)$ is dramatic when $z>12$. This is because the suppression of collapsing is stronger at higher redshift, and weaker at lower redshift. At early universe $z=15$, there is already about $1 \%$ mass in the clouds with size larger than the Jeans length. However, there is only a tiny fraction of mass $\left(\sim 10^{-8}\right)$ in the clouds which are collapsed. This is because at redshift 15 only clouds with mass $>10^{6.5}$ $\mathrm{M}_{\odot}$ can collapse, and hosting star formation. The mass fraction in the collapsed clouds is higher for smaller redshift. Therefore, the formation history of the first-generation baryonic objects lasts the entire epoch from $z=15$ to 10 .

It has been estimated that if all the ionizing photons, and the $\sim 10^{-2}$ metallicity observed at low redshift are produced by the first generation stars, the mass fraction of the IGM falling into the collapsed clouds should not be much less than 1\% (Haiman \& Loeb 1997, Ostriker \& Gnedin 1996) before the reionization. Using the $1 \%$ mass to quantify the first generation of baryonic objects, we can conclude from Table 1 and Fig. 12 that the generation of source responsible for reionization is likely to occur not much earlier than $z=10$, and therefore, the reionization itself should take place not much earlier than that era.

\section{Conclusion and discussion}

We have outlined an evolution picture of the IGM clustering with the LN model, which is capable to sketch cosmic clustering of both the dark matter and the IGM in weakly nonlinear, as well as nonlinear regimes. With the fine numerical simulations, we can make a number of important statistics of the IGM clouds before the reionization. We focus, in particular, the abundance of hydrogen clouds going to collapse, which would be the candidates for the harbor of the first generation stars. While the detail of the first star formation depends on many chemical and hydro factors, the formation of the collapsing candidates is a necessary condition to form stars or galaxies. Therefore, we can set up effective constraint on the history of the formation of the first objects. 
A remarkable feature of the LN model is that the PDF of mass field is long tailed. The long tail events, such as the formation of massive clouds at high redshifts, have a much larger probability than a Gaussian field. The rare events of the first generation collapsed clouds are sensitive to the long tail. For a Gaussian PDF, there are very few events of mass perturbation much larger than $\sigma_{0}$, and therefore, there is no massive objects formed at early time for a Gaussian PDF. However, the LN model predicted a much high probability of forming massive clouds at high redshift.

The LN model provides the density as well as the velocity distributions of the IGM. Comparing the density profile with velocity profile of clouds, one can reveal that clouds with the Jeans mass are not collapsing immediately at high redshift. Typically, the IGM cloud around a group of dark matter potential wells will stay in the phase of slow expanding in proper space for a while. The smaller the mass of hydrogen cloud, the longer the time to remain in this phase. Only clouds with high enough mass can enter the phase of collapsing in proper space. Thus, as shown in Table 1, the minimal mass of hydrogen clouds in collapsing is larger for higher redshifts. That is, the clustering in the LN model is not of the bottom-up hierarchy at scales less than $10^{5-6} \mathrm{M}_{\odot}$. Contrarily, massive collapsed clouds formed in the first, and less massive clouds later. It should be pointed out that this feature cannot simply be explained with the redshift-dependence of the Jeans mass. Although the Jeans mass is smaller at smaller redshift, it is not enough to explain the significant evolution of $M_{\min }$ from $10^{6.5}$ to $10^{4.4} \mathrm{M}_{\odot}$ in the redshift range $15<z<10$. This feature is again because the suppression of the formation of collapsed clouds with small mass on high redshifts. The lognormal mapping makes the evolution of $M_{\min }$ to be much stronger than that of the Jeans mass $M_{J}$.

It should be point out that there are factors leading to overestimate the collapsed hydrogen clouds. First, we count all clouds in the type $3(\S 5.2)$ to be the host of star formation. Obviously, the collapsing clouds in the type 3 are not yet to be that host. Second, the total mass of baryons in the collapsed objects is estimated by the mass of collapsed clouds. This may also be a source of the overestimation. The last but not least, the LN models seems to overestimate the abundance of saturated absorption lines of Ly $\alpha$ (Matarrese \& Mohayaee 2002), it may indicate the excess of dense clouds. Nevertheless, all these factors actually are to strengthen the result on the upper limit to the mass fraction in the first generation objects at a given redshift, and then, to consolidate the conclusion that the generation of source responsible for reionization and the reionization itself is likely to occur not much earlier than $\mathrm{z}=10$.

We may improve the overestimation problem by considering the hydrodynamics of the IGM. However, we would rather like to trade the overestimation with the uncertainty of 
IGM hydrodynamics. That is in the current approach, the conclusions are not dependent on parameters related to the hydro processes of star formation such as cooling time scale, rate of star formation, and efficient of producing reionization photons by stars etc. Our conclusions are, however, sensitively dependent on the power spectrum of initial density perturbations on few $\mathrm{h}^{-1} \mathrm{kpc}$. Therefore, it would be useful to get information of the initial power spectrum on such small scales.

We thank Dr. Ping He and Mr. Hu Zhan for helps during the preparation of this paper. We thank also the referee and editor for their helpful comments.

\section{REFERENCES}

Abel, T., Anninos, P., Norman, M.L. \& Zhang, Y. 1998, ApJ, 508, 518,

Abel, T., Bryan, G. \& Norman M. 2000, ApJ, 540, 39

Bagla, J.S., \& Padmanabhan, T. 1994, MNRAS, 266, 227

Barkana, R., Loeb, A, 2001, astro-ph/0010468

Berera, A. \& Fang, L.Z. 1994, Phys. Rev. Lett., 72, 458

Bi, H.G. 1993, ApJ, 405, 479

Bi, H.G \& Davidsen, A. F. 1997, ApJ, 479, 523.

Bi, H.G., Ge, J., \& Fang, L.Z. 1995, ApJ, 452, 90

Brainerd, T.G., Scherrer, R.J., \& Villumsen, J.V. 1993, ApJ, 418, 570

Bromm, V. Coppi, P.S. \& Larson, R. B. 1999, ApJ, 527, L5

Buchert, T., Dominguez, A. \& Peres-Mercader, J., 1999, A\&A, 349, 343

Cen, R. \& Haiman, Z. 2002, ApJ, 542, L75

Couchman, H.M.P. \& Rees, M.J., 1986, MNRAS, 221, 53

Chiu, W.A. \& Ostriker, J. 2000, ApJ, 534, 507

Eisenstein, D. \& Hu, W. 1998, ApJ, 496, 605

Fang, L.Z., Bi, H.G., Xiang, S.P., \& Börner, G. 1993, ApJ, 413, 477

Feng L.L. \& Fang, L.Z. 2001, ApJ, 535, 519

Feng, L.L. Pando, J. \& Fang, L.Z. 2003, ApJ, 587, 487

Gnedin, N. Y. 2000, ApJ, 542, 535 
Gurbatov, S.N., Saichev, A.I. \& Shandarin, S.F. 1989, MNRAS, 236, 385

Hui, L., Kofman, L. \& Shandarin, S. F., 2000, ApJ, 537, 12

Jamkhedkar, P., Zhan, H. \& Fang, L.Z. 2000, ApJ, 543, L1

Jamkhedkar, P., Feng, L.L., Zheng, W., Kirkman, D., Tytler, D. \& Fang, L.Z. 2003, MNRAS, in press

Jones, B.T., 1999, MNRAS, 307, 376

Kofman, L., Bertschinger, E., Gelb, J.M., Nusser, A., \& Dekel, A. 1994, ApJ, 420, 44

Haiman, Z. \& Loeb, A. 1998, ApJ, 503, 505

Madau, P., 2002, astro-ph/0212555

Madau, P., Meikin, A. \& Rees, M. J. 1997, ApJ, 475, 429

Matarrese, S. \& Mohayaee, R., 2002, MNRAS, 329, 37

Medvigy, D. \& Loeb, A. 2001, astro-ph/0110014

Miralda-Escude, J. 1998, ApJ, 501, 15,

Miralda-Escude, J., Haehnelt, M. \& Rees, M.J., 2000, ApJ, 530, 1

Nusser, A., 2000, MNRAS, 317, 902.

Ostriker, J.P. \& Gnedin, N.Y. 1996, ApJ, 472, L63

Pando, J., Feng, L.L., Jamkhedkar, P., Zheng, W., Kirkman, D., Tytler, D. \& Fang, L.Z. 2002, ApJ, 574, 575

Peebles, P.J.E. 1993, Principles of Physical Cosmology (Princeton: Princeton Univ. Press)

Press \& Schechter 1974, ApJ, 187, 425

Shraiman B.I. \& Siggia, E.D. 2000, Nature, 405, 8

Tegmark, M., Silk, J., Rees, M.J., Blanchard, A., Abel, T., \& Palla, F. 1997, ApJ, 474, 1,

Theuns, T., Schaye, J., Zaroubi, S., Kim, T.-S., Tzanavaris, P., \& Carswell, B. 2002, ApJ, 567, L103

Tozzi, P., Madau, P., Meiksin, A., \& Rees, M. J. 2000, ApJ, 528, 59,

Valageas, P. \& Silk, J. 1999, A\&A, 347, 1

Vergassola, M., Dubrulle, B., Frisch, U. \& Noullez, A. 1994, A\&A, 289, 325.

Vanmarcke, E., 1983, Random Field, (MIT, Mass)

Xu, W., Fang, L.Z. \& Wu, X.P. 2000, ApJ, 532, 728

Yang, X.H., Feng, L.L., Chu, Y.Q. \& Fang, L.Z. 2001, ApJ, 560, 549. 
Zhan, H., Jamkhedkar, P. \& Fang,L.Z. ApJ555, 58

This preprint was prepared with the AAS LATEX macros v5.0. 
Fig. 1. - The Jeans length of baryonic gas (IGM) as a function of the cosmic factor $1 /(1+z)$.

Fig. 2.- The variances of the density fluctuations at the Jeans length as a function of the cosmic scale factor. The dot line is for the linear fluctuation, the solid line is for the non-linear evolution given by the LN model, i.e. $\sigma^{2}=\exp \left(\sigma_{0}^{2}\right)-1$.

Fig. 3.- Volume filling factor (top) and cumulative mass (bottom) as function of the scale factor $a=(1+z)^{-1}$ at redshifts $z=20,12,10$ and 7 in the CDM model.

Fig. 4.- Three samples within $1 \mathrm{Mpc}$ size randomly chosen from $z=15$ (top), $z=$ 10 (middle) and $z=7$ (bottom) simulation. The X-axis is the line-of-sight distance in the comoving space and the Y-axis is the overdensity.

Fig. 5.- Relation between $\rho$ and column density $N_{H I}$ of hydrogen clouds identified from one realization

Fig. 6. - Statistics of hydrogen clouds as function of its column density $N_{H I}$. (a) 1-D differential number density $\left(\mathrm{h} \mathrm{Mpc}^{-1}\right) d N_{c}\left(>N_{H I}\right) / d \ln N_{H I}$; (b) mass fraction of clouds with column density $>N_{H I}$; (c) mean top density $\rho$ and (d) width $\left(\mathrm{h}^{-1} \mathrm{kpc}\right)$. The redshifts are taken to be $z=7$ (solid), 10 (dotted) and 15 (dashed).

Fig. 7.- (a) Hydrogen mass of clouds as a function of column density; (b) cumulated mass friction of clouds with mass $>M$. The redshifts are taken to be $z=7$ (solid), 10(dotted) and 15 (dashed).

Fig. 8. - 3-D number density of clouds as functions of (a) column density $N_{H I}$, and (b) mass $M$ of clouds. The redshifts are taken to be $z=7$ (solid), 10(dotted) and 15 (dashed).

Fig. 9.- Density profiles of clouds at redshifts 15, 12, 10 and 7. For each redshift, the successive curves from lower to higher correspond to column density $\log N_{H I}=16.25+m \times$ 0.5 , and $m=0,1, \ldots 8$.

Fig. 10.- Velocity profiles of clouds at redshifts 15, 12, 10 and 7. For each redshift, the successive curves from lower to higher correspond to column density $\log N_{H I}=16.25+m \times$ 0.5 , and $m=0,1, \ldots 8$.

Fig. 11. - The same as Fig. 11, but for central parts of clouds.

Fig. 12. - The cumulative mass fraction of $M\left(>M_{J}\right)$ and $M\left(>\rho_{\min }\right)$ as function of redshift. 


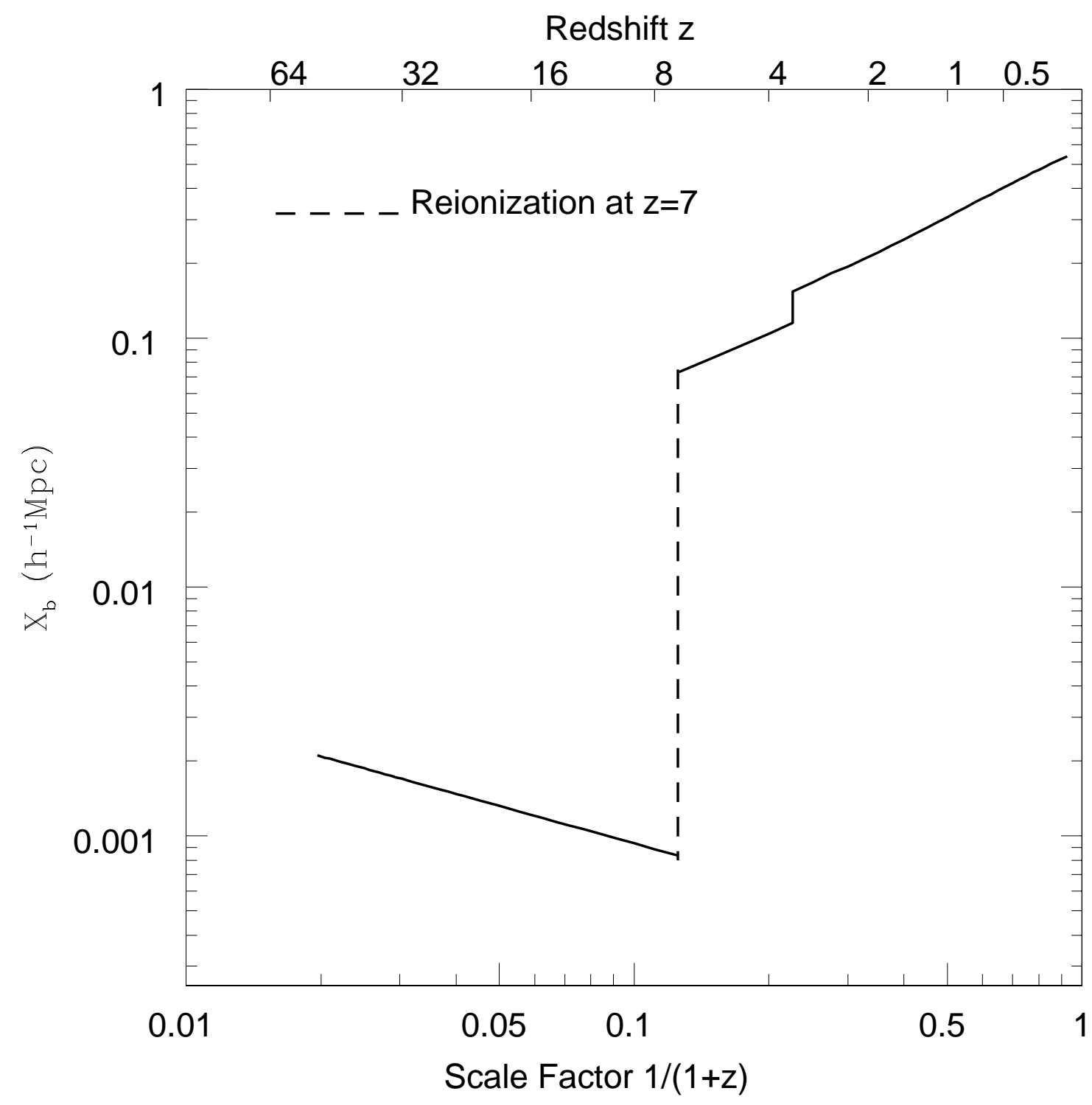




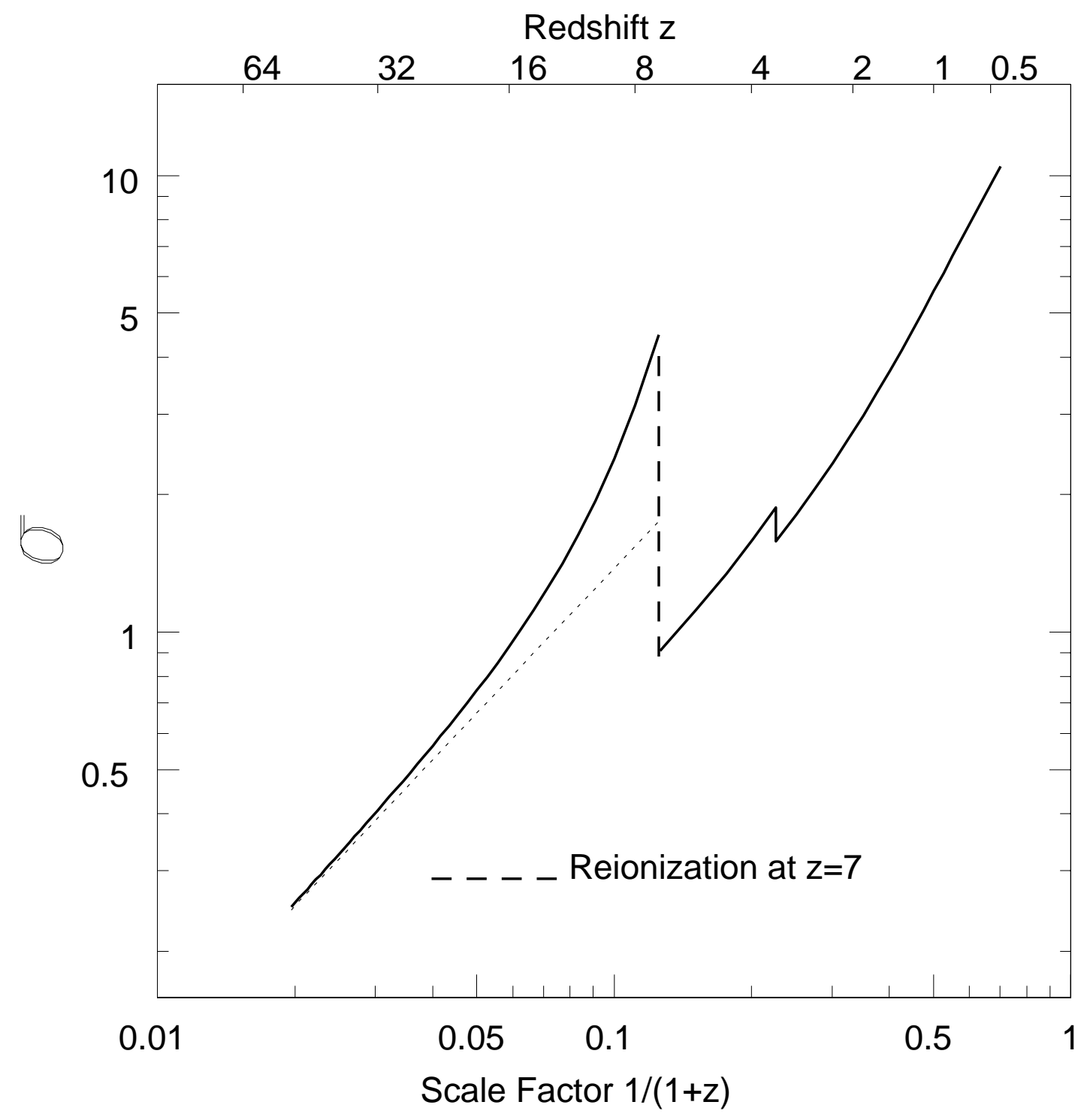



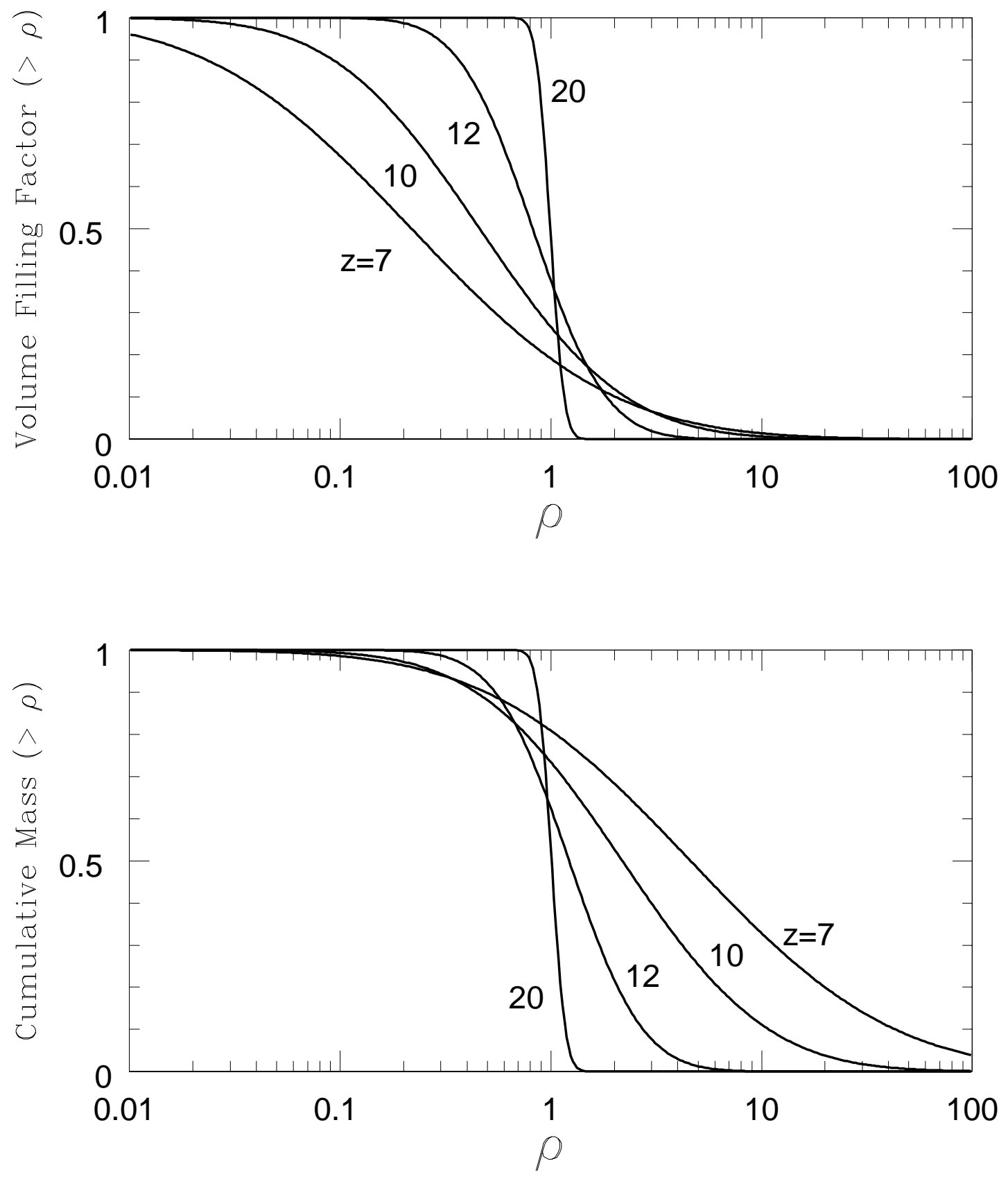


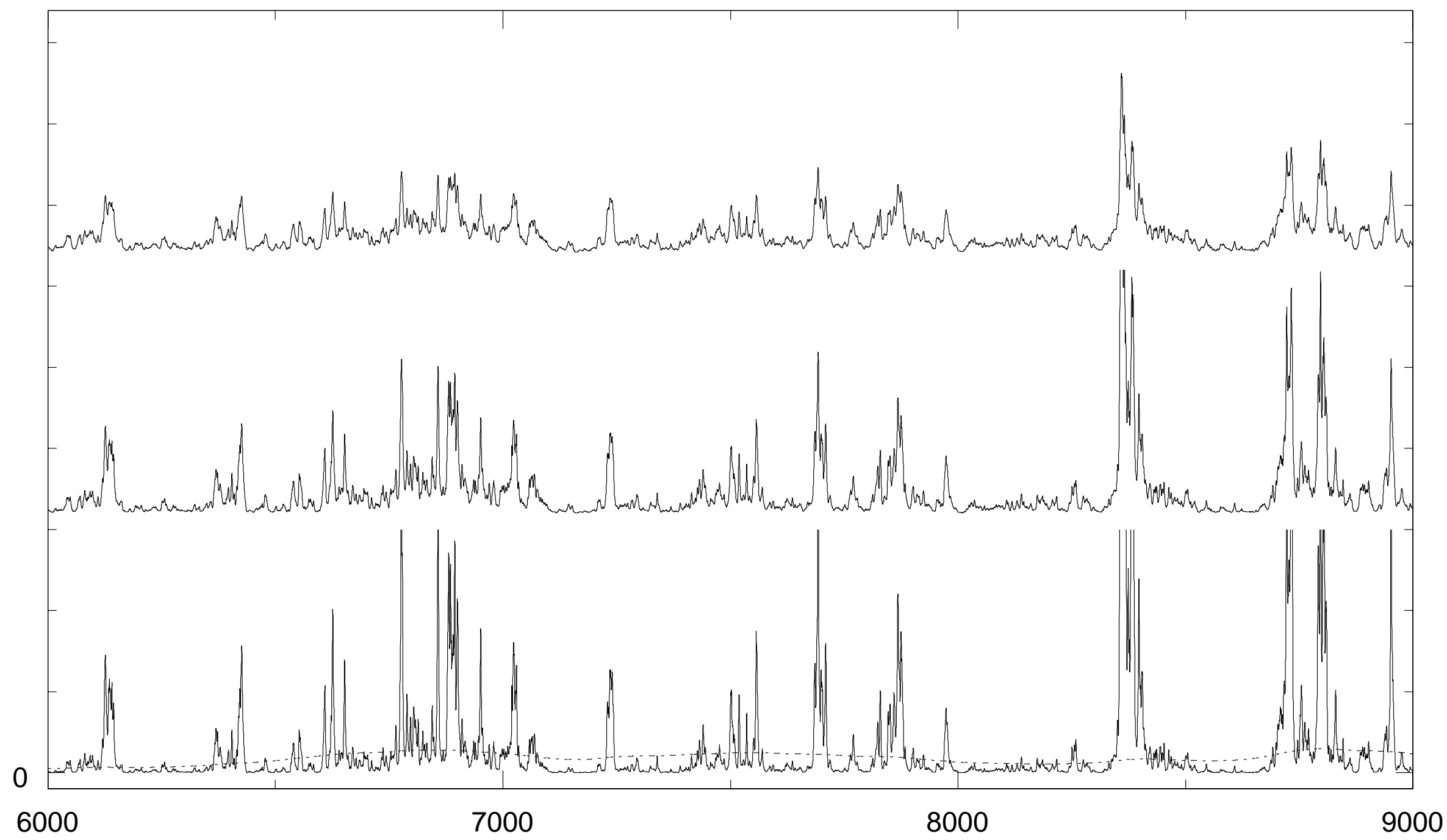




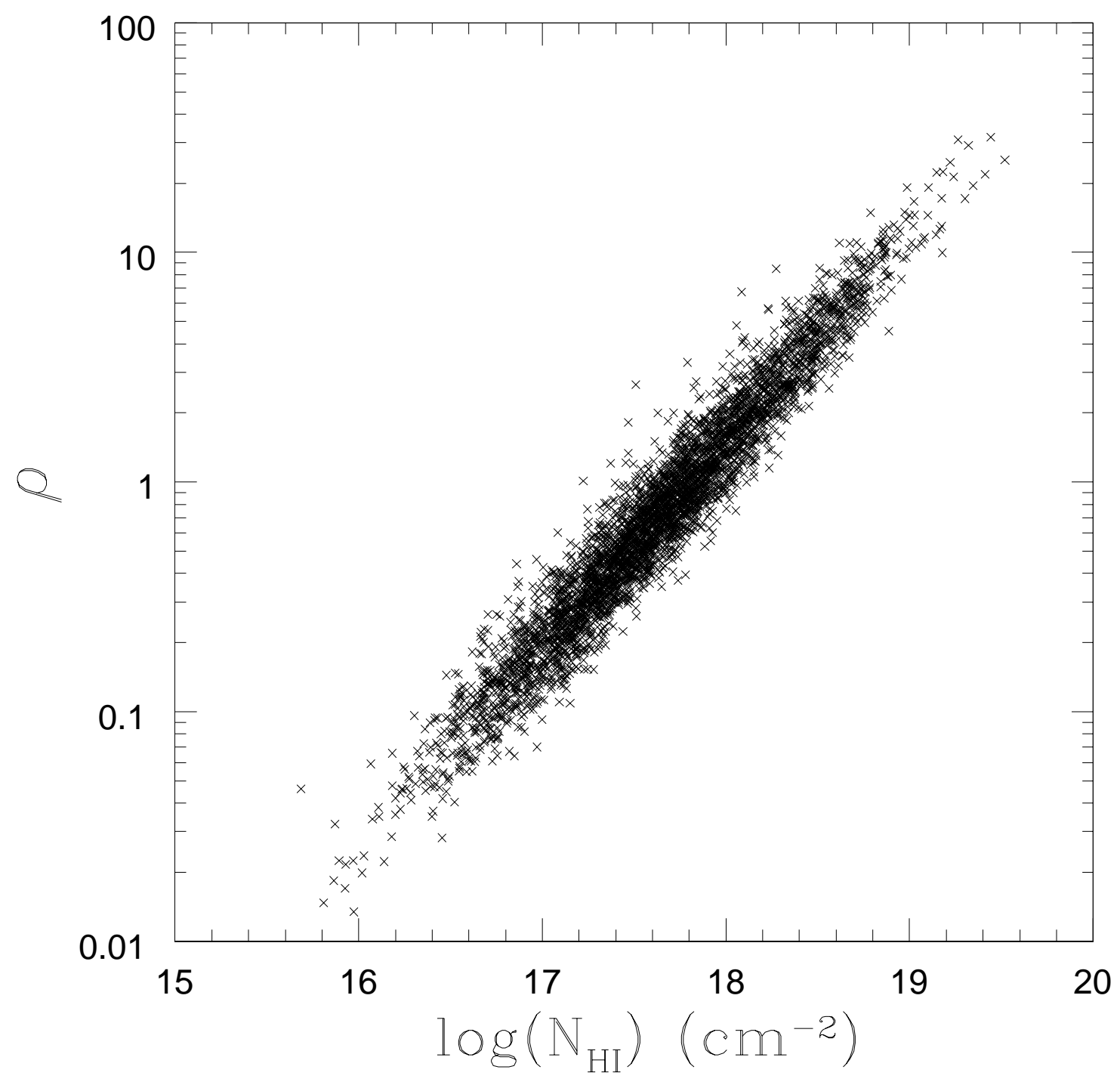



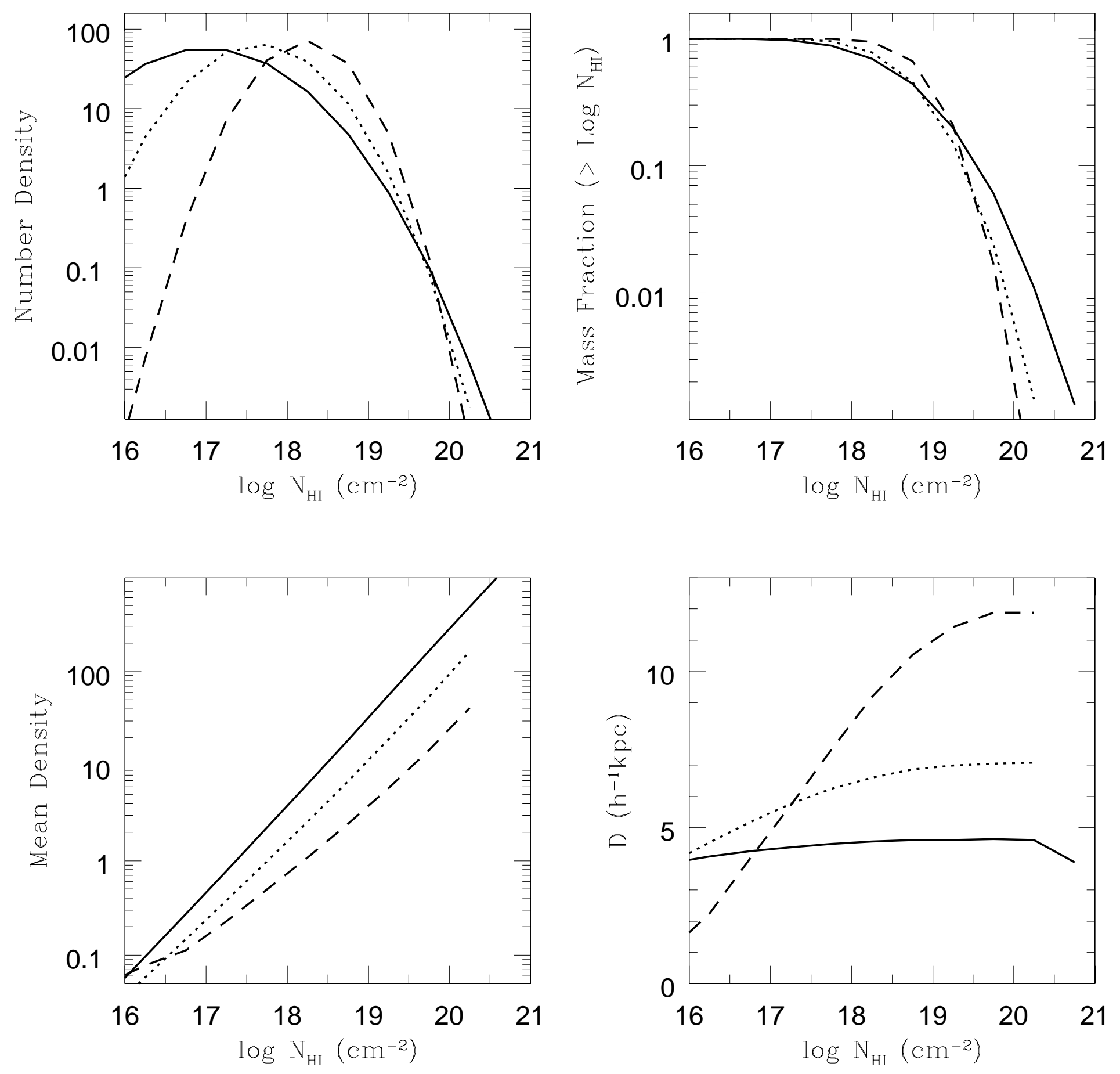

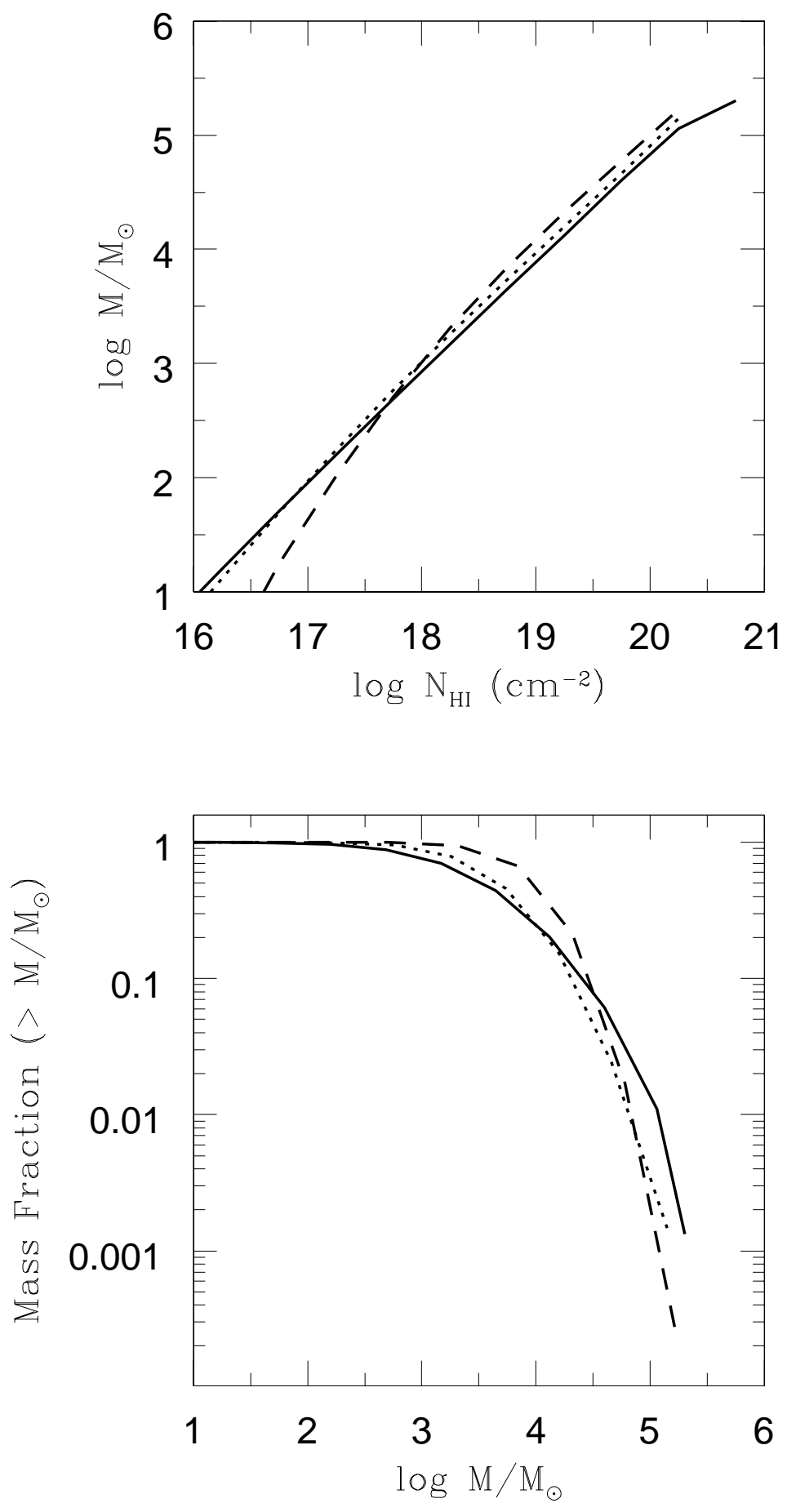

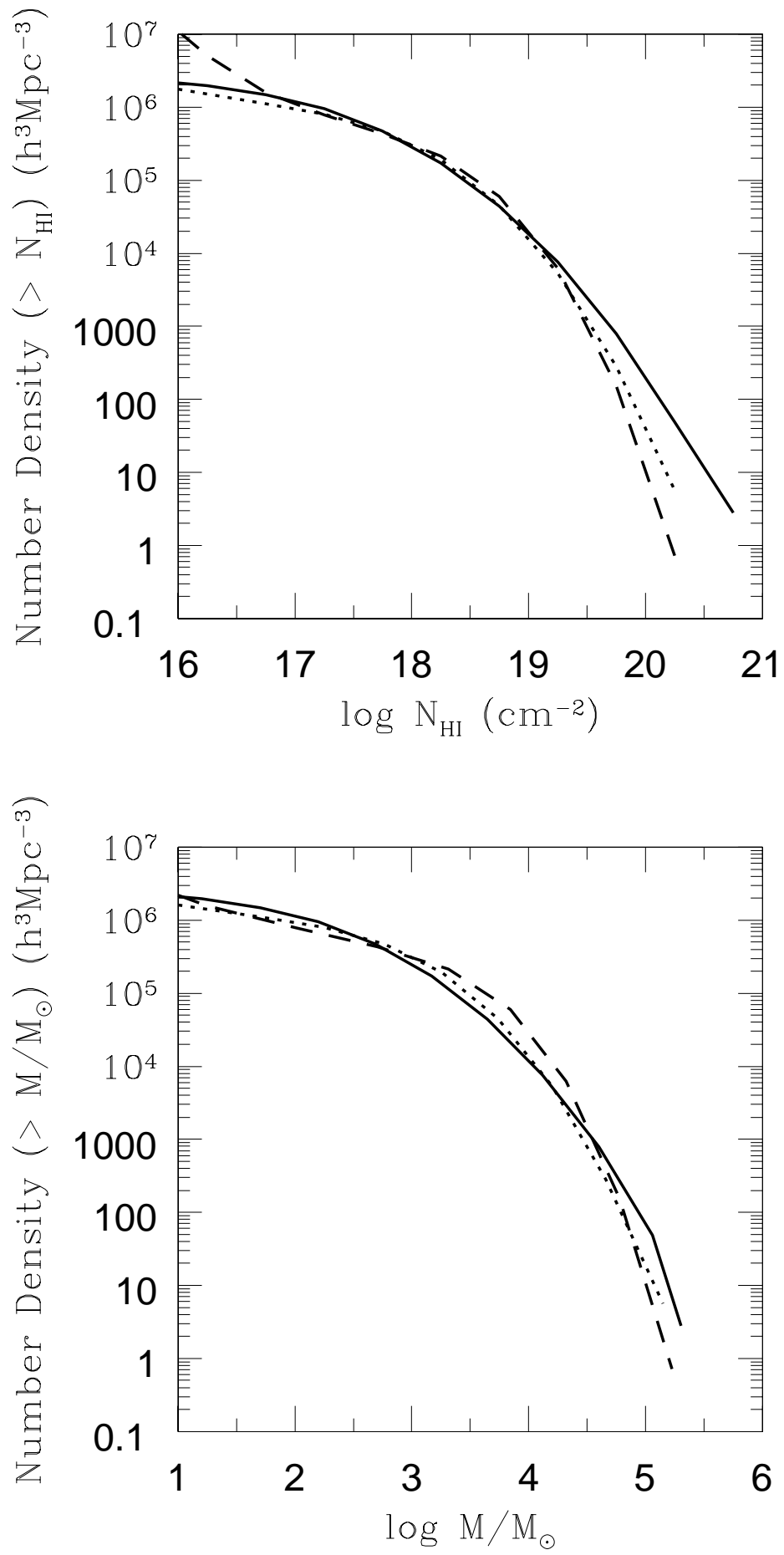

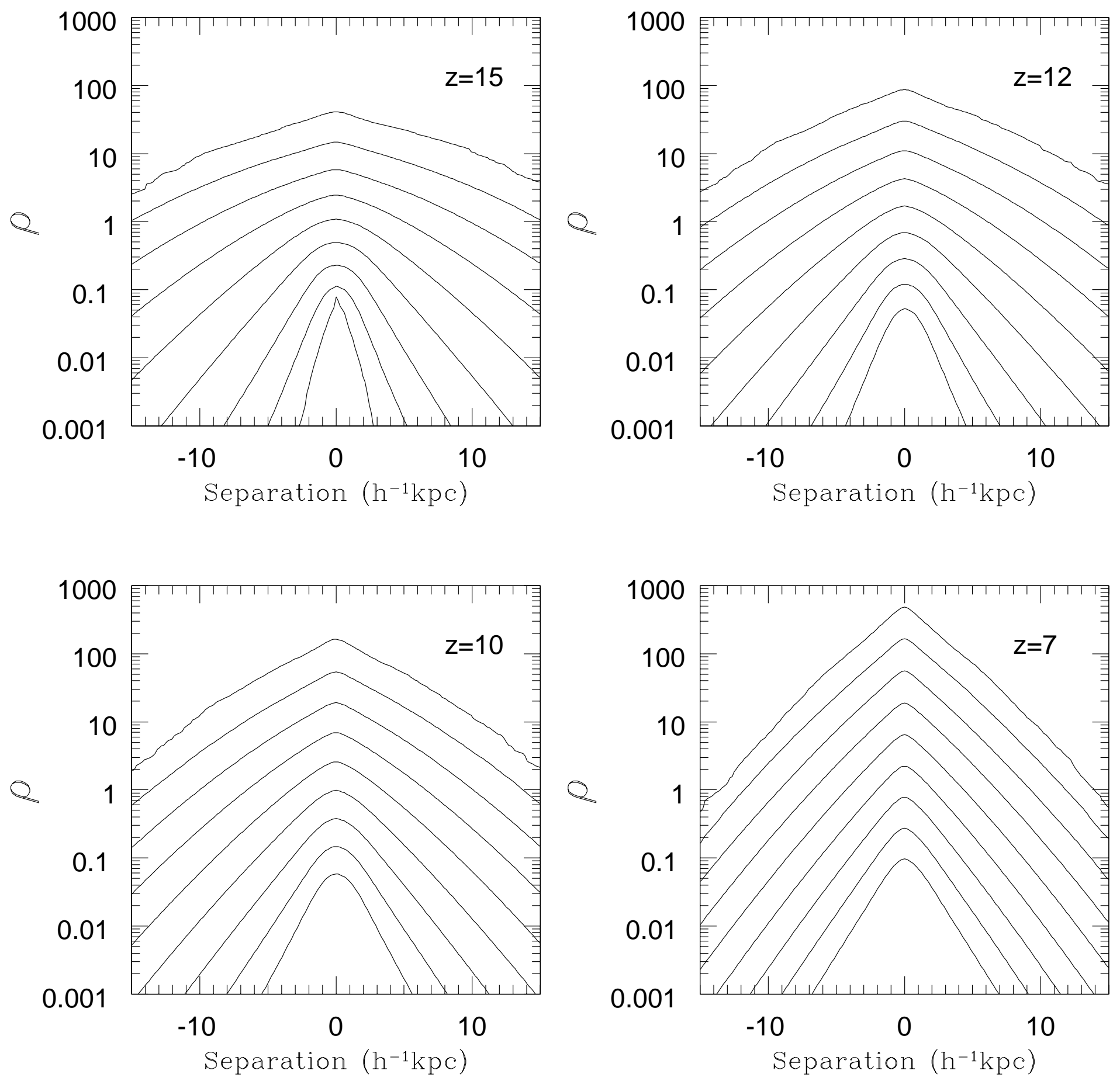

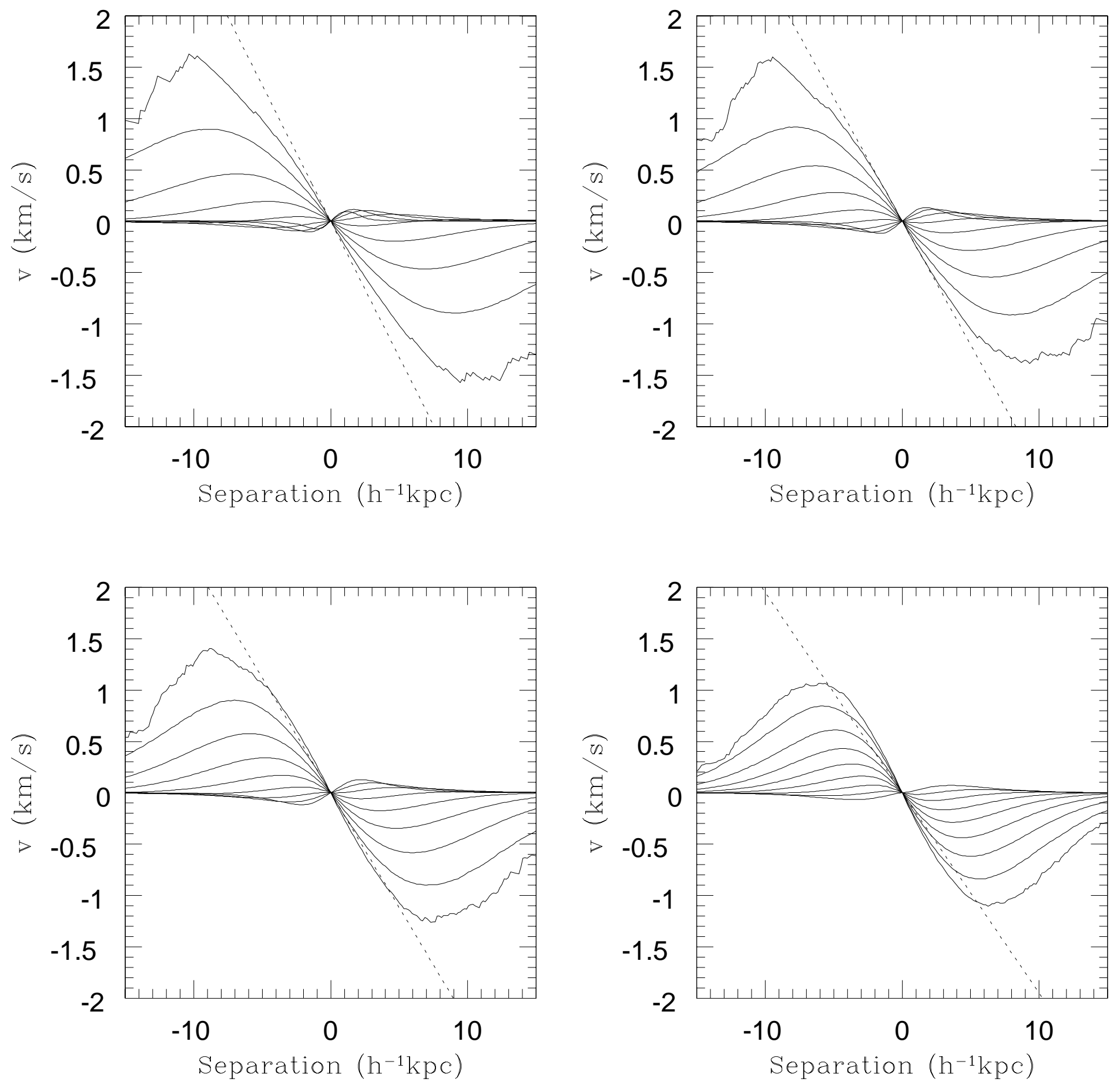

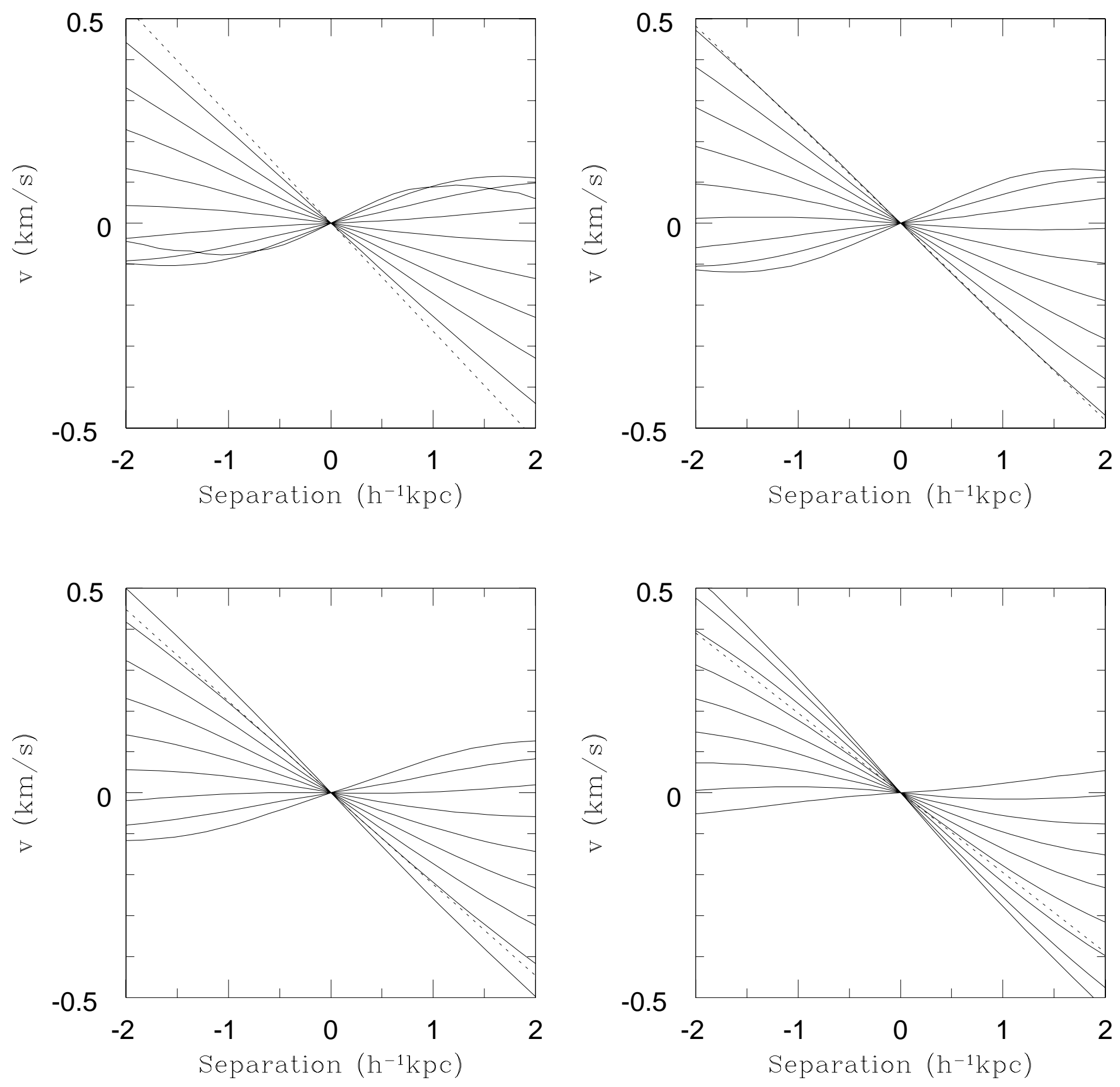


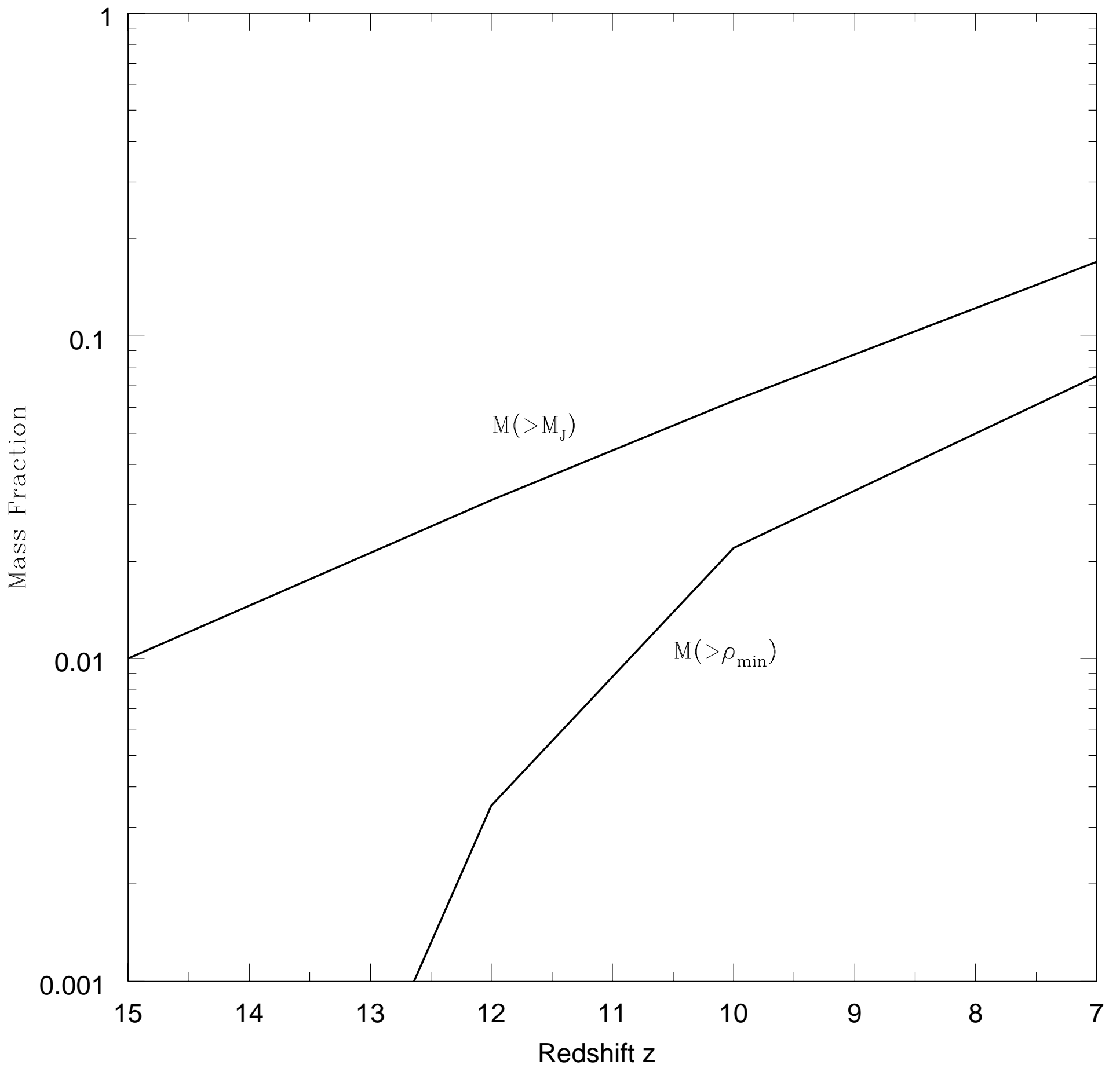

